\title{
Event conjunction: How the hippocampus integrates episodic memories across event boundaries
}

\author{
Benjamin J. Griffiths ${ }^{1}$ \& Lluís Fuentemilla ${ }^{2}$
}

1) School of Psychology, University of Birmingham, Birmingham, UK

2) Institute of Neurosciences, University of Barcelona, Barcelona, Spain

Contact Information

Benjamin J. Griffiths

b.j.griffiths.1@pgr.bham.ac.uk 


\begin{abstract}
Our lives are a continuous stream of experience. Our episodic memories, however, have a definitive beginning, middle and end. Theories of event segmentation suggest that salient changes in our environment produce event boundaries which partition the past from the present and, as a result, produce discretised memories. However, event boundaries cannot completely discretise two memories; any shared conceptual link will eagerly integrate these memories. Here, we present a new framework inspired by electrophysiological research that resolves this apparent contradiction. At its heart, the framework proposes that hippocampal theta-gamma coupling maintains a highly abstract model of an ongoing event and serves to encode this model as an episodic memory. When a second but related event begins, this theta-gamma model is rapidly reconstructed within the hippocampus where new details of the second event can be appended to the existing event model. The event conjunction framework is the first electrophysiological explanation of how event memories can be formed at, and integrated across, event boundaries.
\end{abstract}

\title{
Episodic memories are formed at event boundaries
}

Life is a continuous stream of experience. Episodic memories, however, are discretised short stories of our personal past, anchored to a singular point in time and space (Tulving, 2002). Somewhere between the forming and retrieving of these memories, therefore, a process must arise to carve unique memories from this continuous experience. Numerous cognitive theories set out to address this very question. Event segmentation theories (Radvansky, 2012; Zacks \& Swallow, 2007) state that when a salient change in environment occurs, an event boundary is created. The event boundary is thought to segregate the past and present, and induce a rapid and retroactive encoding of experience prior to the boundary to produce a discretised episodic memory (Radvansky \& Zacks, 2017). Other accounts suggest that elements of an event are proactively encoded during the course of an event through shared contextual links (Clewett, Dubrow, \& Davachi, 2019; Schapiro, Rogers, Cordova, TurkBrowne, \& Botvinick, 2013). When an event boundary is encountered, it produces a large contextual shift that prevents the formation of contextual link between elements of the new event and those of the previous event. While the proactive and retroactive accounts have their differences, they all agree that the discretisation of event memories (i) occurs during encoding, and (ii) hinges upon the presence of event boundaries.

Event boundaries can take many forms, including temporal boundaries (DuBrow \& Davachi, 2013, 2016; Ezzyat \& Davachi, 2011), spatial boundaries (Horner, Bisby, Wang, Bogus, \& Burgess, 2016), and goal-related boundaries (Speer, Zacks, \& Reynolds, 2007; Zacks et al., 2001). Importantly, all these forms of boundary exert influence over episodic memory. For example, Horner and colleagues (2016) asked participants to navigate a series of virtual rooms, each of which contained multiple objects, and then tested the participants ability to recall the sequence of objects within a room (where there was no event boundary) and between rooms (where the door introduces a spatial boundary into the sequence; Radvansky \& Copeland, 2006). Participants showed a significant impairment when trying to recall object sequences that crossed spatial boundaries relative to sequences contained within one room. These results suggest that episodic memory is organised in accordance with the spatial boundaries that participants encountered. Ezzyat and Davachi (2011) conceptually replicated this effect by using temporal boundaries (i.e. time jumps in short narratives) in place of spatial boundaries. Interestingly, individuals who are more effective at segmenting events recall many more details about these events, even after controlling for a variety of cognitive and demographic confounds (Sargent et al., 2013). As such, it seems that the presence and detection of event boundaries is critical to the successful formation of episodic memories. 
On a neuroanatomical level, event segmentation employs a hierarchical network to detect boundaries (Baldassano et al., 2017); low level sensory regions are sensitive to fine-grained changes in events while high level multimodal regions detect coarse and abstract shifts. In one of the first neuroimaging studies of event segmentation, Zacks and colleagues (2001) time-locked functional magnetic resonance imaging (fMRI) data to event boundaries present in video clips that depicted everyday tasks. Analysis revealed a strong increase in the activity of the posterior medial cortex at these boundaries relative to epochs just before these boundaries. Speer and colleagues (2007) conducted a similar study where narrative boundaries were used in place of perceptual boundaries and they, again, found boundary-related neural activity in the posterior medial cortex (mostly greatly in the precuneus). Notably, activation in the precuneus was greater for coarse event boundaries (i.e. great shifts in environment) relative to fine-grained event boundaries (e.g. minor changes in an ongoing task). Further elaborating upon this apparent selectivity to coarse boundaries, Baldassano and colleagues (2017) built a hierarchical model of event structure using multivariate pattern analysis. They demonstrated that areas in the posterior medial cortex are responsive to multi-modal, abstract boundaries (corroborating the findings of Speer and colleagues), while sensory regions (such as the early visual cortex) are more responsive to fine-grained boundaries. Together, these experiments provide strong support to suggest that the brain can detect event boundaries through a hierarchical network. How, though, does the brain use these boundaries to form an event memory?

To form an event memory, all constituent elements of an event need to be bound together to form a coherent representation. The hierarchical network aids in the detection of event boundaries that define where an event begins and ends, but the network does not aid in the association of elements within the event. Rather, this task is thought to fall on the hippocampus (Konkel \& Cohen, 2009; Olsen, Moses, Riggs, \& Ryan, 2012; Wallenstein, Eichenbaum, \& Hasselmo, 1998). Numerous studies indicate that the hippocampus is a relational hub that associates distinct, abstract concepts (Davachi, 2006; Staresina \& Davachi, 2009). As such, it is well positioned to associate distinct elements into a singular event representation. In line with this idea, Schapiro and colleagues (2016) demonstrated that item representations within the hippocampus share greater representational similarity with items that shared a temporal context relative to items that did not, suggesting that the hippocampus can generate links between distinct elements occurring in the same context. Intriguingly, the hippocampus is also linked to the maintenance of recently encountered stimuli (Axmacher et al., 2010; Piekema, Kessels, Mars, Petersson, \& Fernandez, 2006), suggesting it could retain early elements of an event while later elements of the event unfold. Together, one can speculate that the hippocampus creates a model of an ongoing event by maintaining the distinct elements of the event (e.g. Axmacher, Henseler, et al., 2010) and linking these elements together (Schapiro et al., 2016; Staresina \& Davachi, 2009) into a coherent event representation.

Beyond forming and maintaining relational links, a cornucopia of studies have demonstrated that the hippocampus plays a critical role in the formation of episodic memories (e.g. Corkin, 2002; Davachi, 2006; Giovanello, Schnyer, \& Verfaellie, 2009; Nyhus \& Curran, 2010). As such, one could further speculate that the hippocampus is pivotal in transforming the aforementioned event models into an episodic memory. Testing this idea, Ben-Yakov and Dudai (2011) investigated hippocampal function during the formation of event memories. The hippocampus showed a strong response to the offset of the video clip regardless of whether the clip was remembered or not (similarly found in Baldassano et al., 2017). Critically however, the hippocampal response to remembered clips was significantly greater for remembered items relative to forgotten items, indicating that this boundary response was linked to memory formation. A number of control analyses concluded that this effect was specific to the boundary induced by video offset, rather than video duration or the contents of the videos. The authors speculate that these responses reflect the rapid replay of the event to solidify its memory 
trace. In line with this idea, two EEG studies (Silva, Baldassano, \& Fuentemilla, 2019; Sols, DuBrow, Davachi, \& Fuentemilla, 2017) found that neural patterns relating to an event are replayed within a second of encountering an event boundary, and that this replay is predictive of later memory. Whether this replay originates from the hippocampus (as in rodents; Carr, Jadhav, \& Frank, 2011), however, is an open question.

In sum, there is strong evidence to suggest that event boundaries serve to segregate the past and present, and create discrete episodic memories. While a large hierarchical network appears to be involved in the processing of event boundaries, it seems that the hippocampus is the region best suited for the maintenance of the event model, and the transference of this model to episodic memory. It is worth noting, however, that the idea that event boundaries provide a definitive start and end of a memory does not easily reconcile with decades of research into reconsolidation, memory integration and retrieval-induced learning - all of which show that episodic memories are malleable and subject to updating based on later information.

\section{Episodic memories are integrated across event boundaries}

If two events separated in time share a common goal and/or location, what happens to the two event memories? Evidence suggests they become linked in such a way that the retrieval of one brings the other to the forefront of memory (e.g. Griffiths, Mazaheri, Debener, \& Hanslmayr, 2016; Miller et al., 2013). Can it really be concluded, therefore, that episodic memories are definitively orthogonalised by event boundaries? Here, we ask how temporally-discontinuous events, separated by event boundaries, have the potential to be fused together.

If two memories share a commonality, they can become integrated. This has been widely demonstrated in associative inference paradigms, where participants are presented with stimulus pairs $A B$ and $B C$ and then probed to see if they can make an inferential link between $A$ and $C$ (which have never been presented together). Any knowledge of link between $A$ and $C$ would suggest that the distinct memory traces $A B$ and $B C$ have become integrated. Evidence suggests humans are remarkably adept at such inferential tasks (e.g. Backus, Schoffelen, Szebényi, Hanslmayr, \& Doeller, 2016; Schlichting, Zeithamova, \& Preston, 2014; Tompary \& Davachi, 2017; Zeithamova, Dominick, \& Preston, 2012). How two memories become integrated, however, is still open to debate. Some theories propose that integration occurs during the encoding of the second memory (i.e. integrative encoding; Nadel, Hupbach, Gomez, \& Newman-Smith, 2012; Sekeres, Winocur, \& Moscovitch, 2018), while others propose that integration occurs during consolidation or retrieval (i.e. offline integration; Polyn, Norman, \& Kahana, 2009; Tompary \& Davachi, 2017). These processes are not mutually exclusive however, meaning memory integration could theoretically occur both during encoding and during offline periods (Zeithamova \& Preston, 2010). Here, we concern ourselves with integrative encoding (for further details on offline integration and event memories, see Clewett, Dubrow, \& Davachi, 2019).

On a neural level, memory integration has been proposed to rely on the coordinated activity of the hippocampus and the medial prefrontal cortex (mPFC; Schlichting \& Preston, 2015). In this division of labour, the hippocampus is thought to encode details of unfolding events and reactivate details of past events, while the MPFC is thought to find commonalities across episodes and bias hippocampal reactivation towards relevant past events (Preston \& Eichenbaum, 2013). In line with these predictions, patients with lesions either to the mPFC (Spalding et al., 2018) or the hippocampus (Pajkert et al., 2017) suffer deficits in the memory integration that cannot be explained by deficits in associative memory. As the nature of these lesions did not change between encoding and inference, however, these studies do not clarify whether these regions specifically impair integrative encoding. 
To address this, Schlichting and colleagues (2014) contrasted hippocampal activation during associative encoding (i.e. the first pair; $A B$ ) and integrative encoding (i.e. the second pair; $B C$, where integration of $A$ and $C$ can occur). They observed an increase in hippocampal activity during successful integrative encoding relative to successful associative encoding, indicating that the hippocampus plays a role in the online integration of episodic memories. Taking a similar approach to MEG, Backus and colleagues (2016) produced complementary results indicating that increases in hippocampal power are predictive of integration. Moreover, Backus and colleagues also uncovered evidence to suggest that connectivity increases between the hippocampus and medial prefrontal cortex (mPFC) during online integration, conforming to the idea that a hippocampal-mPFC circuit underpins the online integration of distinct episodic memories. The mechanistic interpretation of these two results remains open to debate. Under the framework proposed by Schlichting \& Preston (2015), one would speculate that the hippocampal-mPFC connectivity reflects the directed reactivation of the original (i.e. AB) pair. However, the hippocampal activation may also reflect representational binding between the first and second pairs (Davachi, 2006; Staresina \& Davachi, 2009). Indeed, one could speculate that the activation observed here is a blend of both.

Current theories of event segmentation do not address how two highly overlapping event memories interact. The studies discussed here, however, suggest that a cue in the current environment can lead the reactivation of a previously encoded memory, which can be associated with an ongoing event. One could therefore speculate that previously-encoded events relevant to current experience may be reactivated and integrated with the current event model to provide a more comprehensive and complete event memory. While the cornerstone AB-BC memory integration paradigm is highly abstract and lacks the ecological validity present in event segmentation memory paradigms, event boundaries still exist in the memory integration paradigm (i.e. the end of a trial).

\section{A role for neural oscillations}

Rhythmic fluctuations in neural activity are referred to as neural oscillations. Numerous empirical studies have demonstrated that the synchronisation of theta $(3-7 \mathrm{~Hz})$ and gamma $(30-100 \mathrm{~Hz})$ oscillations relate to successful episodic memory formation (for review, see Colgin, 2015; Hanslmayr, Staresina, \& Bowman, 2016; Nyhus \& Curran, 2010). An increase in the synchronisation of hippocampal gamma oscillations is thought to facilitate spike-timing-dependent plasticity (STDP; Axmacher, Mormann, Fernández, Elger, \& Fell, 2006; Nyhus \& Curran, 2010) - a form of long-term potentiation (LTP) that depends on the highly precise firing of presynaptic and postsynaptic neurons. Probing rat hippocampal neurons in vitro, Bi and Poo (1998) showed that the postsynaptic neuron must fire $20 \mathrm{~ms}$ (i.e. $50 \mathrm{~Hz} /$ gamma) after the presynaptic neuron to induce LTP. Given that these hippocampal neurons have been shown to lock gamma-band activity (Jutras, Fries, \& Buffalo, 2009) and gamma-band activity is predictive of memory formation (e.g. Long \& Kahana, 2015), one could speculate that increases in the amplitude of hippocampal gamma oscillations reflect increases in STDP.

In regards to theta activity, the phase of the oscillation has been thought to dictate whether LTP or long-term depression (LTD) occurs (Huerta \& Lisman, 1995; Hyman, Wyble, Goyal, Rossi, \& Hasselmo, 2003; Pavlides, Greenstein, Grudman, \& Winson, 1988). This has led to the suggestion that the peak of the theta cycle is optimal for memory encoding while the trough of the cycle is optimal for memory retrieval (Hasselmo, 2005). Indeed, computational models implementing this principle demonstrate how the hippocampus can effectively encode new temporal sequences and retrieve existing sequences without incurring catastrophic interference between the two processes (Schapiro, TurkBrowne, Botvinick, \& Norman, 2017). Evidence that supports the idea of such a mechanism in humans is, however, incomplete. Nevertheless, pieces of the puzzle have been revealed. For example, Clouter 
and colleagues (2017) demonstrated that two stimuli presented at the same phase of theta are more likely to be successfully encoded than two stimuli that presented at opposing phases, indicating that associative memory formation varies as a function of theta phase. Moreover, Kerrén and colleagues (2018) demonstrated that neural evidence (as measured using multivariate pattern analysis) for retrieved stimuli fluctuate at approximately $7 \mathrm{~Hz}$, suggesting that episodic memory retrieval is also dependent on theta phase.

While the origins and proposed functions of hippocampal theta and gamma rhythms are distinct, gamma oscillations are frequently seen to nest within the ongoing theta cycle (Colgin \& Moser, 2010) - a phenomenon known as theta-gamma phase-amplitude coupling. Numerous studies have demonstrated that theta-gamma coupling correlates with successful memory formation (Griffiths et al., 2018; Heusser, Poeppel, Ezzyat, \& Davachi, 2016; Staudigl \& Hanslmayr, 2013; Tort, Komorowski, Manns, Kopell, \& Eichenbaum, 2009). Phase-amplitude coupling is thought to provide a neural code capable of recording sequences (Lisman \& Jensen, 2013). Under this framework, each gamma cycle is thought to reflect the firing of a cell population that codes for a unique element in the sequence. Meanwhile, the theta cycle organises the sequence, dictating the order in which elements are encoded. In a test of this theory, Bahramisharif and colleagues (2018) asked participants to retain a sequence of three letters for several seconds and examined whether gamma-band representations of these letters peaked at distinct phases of the theta cycle. Indeed, they found that gamma-band representations of the first letter peaked earlier in the theta cycle than the second letter, and representations of the second letter peaked earlier in the theta cycle than the third letter. This result demonstrates that theta-gamma phase-amplitude coupling can provide a neural framework that codes for sequences of stimuli. Heusser and colleagues (2016) expanded this finding to episodic memory by asking participants to encode a sequence of images and then testing their knowledge of the sequence. Theta-phase locked gamma power increased with the addition of each item to a sequence, and critically, the phase at which gamma power locked became progressively later for each successive item, indicating that every item was added to the end of the existing theta-gamma code. Importantly, it would appear that theta-gamma coupling not only provides a method to link together elements of an event, but also provides a method to maintain these elements for the duration of the event (Axmacher et al., 2010). Together, these results demonstrate that hippocampal theta-gamma phase-amplitude coupling can organise and maintain a neural code capable of encoding sequences into episodic memory.

The proposed neural code supported by hippocampal theta-gamma coupling may appear vastly different to the proposed roles of hippocampal theta and gamma in isolation. However, the principles can be reconciled. Gamma oscillations within a theta-gamma code ensure that cell populations coding for each element fire approximately $20 \mathrm{~ms}$ after the preceding cell population fires for the preceding element. As discussed above, this temporally precise firing allows STDP to strengthen the synaptic connections between two cell populations and, in the case of a theta-gamma code, would create a chain of elements (first to second element, second to third element etc.). In other words, the ability for gamma oscillations to facilitate STDP makes it ideal for creating temporally-sequenced memories. A notable limitation of this view is that, in isolation, there is no mechanism to terminate such a sequence. Theta phase-dependent plasticity remedies this: as gamma rides the peak of the theta cycle, a sequence can be generated through gamma-linked STDP (Bi \& Poo, 1998) and further enhanced by theta-related LTP (Huerta \& Lisman, 1995; Pavlides et al., 1988). During the trough of the theta cycle however, gamma-related increases in STDP could become negated by theta-related LTD, terminating any associative change generated by gamma oscillations. A recent computational model implementing this concept has demonstrated that the combination of theta and gammarelated LTP is an effective method to form associative memories (Parish, Hanslmayr, \& Bowman, 


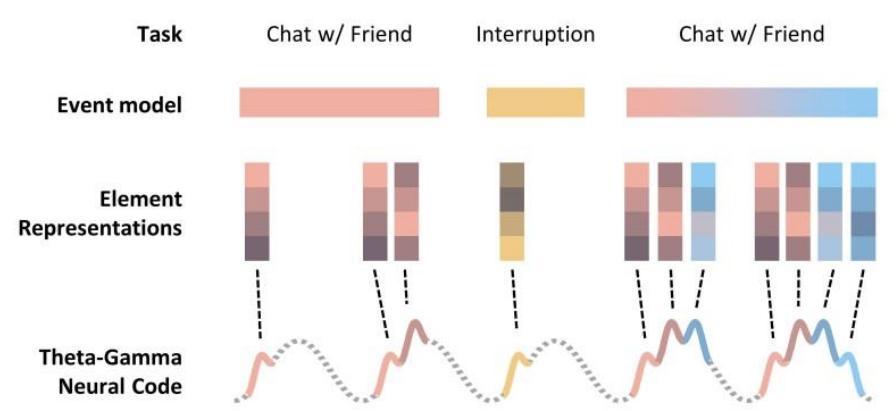

Figure 1. The event conjunction framework. Event boundaries (e.g. answering a phone call) segment a continuous stream of experience into discrete events. These discrete events are represented within the brain as event models. Event models consist of a combination of 'elements' (e.g. time, space, goals). As the event unfolds, more elements are added to the model. We propose that theta-gamma coupling within the hippocampus supports these event models by maintaining the temporal sequence of these elements and steadily encoding the event model through long-term potentiation. At an event boundary, the current event model is replaced by a new model. The same happens to the theta gamma code. When a boundary contains a cue to a previous event, that event is rapidly reactivated and subsequently updated with new elements relating to the new event.
2018). Therefore, hippocampal thetagamma coupling may be not only be the most effective method for maintaining/representing sequences (Lisman \& Jensen, 2013), but also for encoding these sequences as episodic memories.

\section{The case for event conjunction}

So far, we have discussed how (i) event segmentation dictates the formation of episodic memories, (ii) these memories can be integrated across event boundaries, and (iii) hippocampal thetagamma coupling may provide a mechanism to maintain and encode episodic memories. Here, we entwine these threads to provide a new view on how episodic memories are formed through the process of event segmentation.

Take the following example: you are in a café with an old friend who is telling

you a story about their new boss. You are interrupted by an urgent phone call so you step outside to answer, introducing a change in location and in goal. You then return to your friend, who resumes their story. This example includes two event boundaries, marked by leaving to answer the call and then returning to the café interior. Existing theories of event segmentation would therefore predict that three distinct memories would be formed; (i) the details of the story told prior to the phone call, (ii) the details of the phone call, and (iii) the details of the story told after the phone call. Intuitively however, you are likely to recall the friend's story in its entirety despite the event boundaries that occurred between the telling of the story. In other words, integration has arisen to conjoin the two related events into a singular coherent memory. Indeed, we may further intuit that this integration would occur as the third event unfolds, because details from the first event (i.e. the beginning of the story) are essential to comprehending the details of the remaining story. We refer to the idea that two overlapping events can be integrated into a singular memory as 'event conjunction'.

We propose that theta-gamma phase-amplitude coupling within hippocampus lies at the heart of event conjunction. During the course of the first event, each element of the ongoing event is coded for by a unique hippocampal cell population locked to a discrete phase of the ongoing theta cycle (Bahramisharif et al., 2018; Lisman \& Jensen, 2013). This event model is maintained within the hippocampus as a theta-gamma code for the duration of the event (Axmacher et al., 2010). As the event unfolds and becomes more complex, new cell populations coding for new elements of the event are added to the end of the existing theta-gamma code (Heusser et al., 2016). The propensity for hippocampal theta-gamma coupling to induce LTP ensures that the ongoing event is steadily and proactively encoded as it unfolds. When an event boundary is encountered, the code is rapidly replayed to facilitate additional retroactive encoding of the finalised event representation (Sols et al., 2017). This could be reflected in a spike in hippocampal activity after the boundary (Baldassano et al., 2017; Ben-Yakov \& Henson, 2018). When another event boundary is encountered containing a cue to 
the previously encoded event, the guided reactivation of the earlier event arises through interactions between the hippocampus and medial prefrontal cortex (Backus et al., 2016; Schlichting \& Preston, 2015). Detailed information about the event is reinstated in the neocortex and then circled back to the hippocampus, where the theta-gamma code is recreated (Koster et al., 2018). Elements of the ongoing event are then appended to the recreated theta-gamma code in the same way in which new elements were added to the code during the unfolding of the original event. As a result, the current event becomes conjoined with the original event.

A number of key predictions can be derived from this framework, each of which is discussed below:

1) Events have an optimal duration. Every theta cycle sees a repetition of the event's thetagamma code, and hence every repetition sees further strengthening of the event representation through LTP. Longer events, therefore, undergo more LTP than shorter events and hence will be better encoded. This is by no means a controversial prediction, as numerous studies have demonstrated that additional rehearsal time sees a boost in memory performance (e.g. Craik, 1970; Greene, 1987; Meunier, Ritz, \& Meunier, 1972). Importantly however, overly long events may also be difficult to encode as they contain too many unique elements and hence cannot be maintained by a hippocampal theta-gamma code. As such, our framework would predict that there is an optimal duration for events; overly short events suffer too little LTP to be encoded, while overly long events become too complex to be encoded. The optimal duration of an event, though, remains a mystery.

2) Details of overly long events are more likely to suffer interference than details of shorter events. Long events with a large number of elements would require a large number of gamma cycles to fit onto the ongoing theta cycle. At some point, however, physiological constraints are encountered where no further gamma cycles can be appended to the theta cycle. One could therefore speculate that some elements of overly long events cannot be encoded. In support of this idea, evidence suggest that memory performance declines as the number of items to encode increases (Unsworth \& Engle, 2006). What happens when this limit is reached is an open question however. Are later elements less likely to be remembered as they cannot be added to the existing theta-gamma event model? Are insignificant (or weakly represented) elements discarded from the theta-gamma code in favour of later, more relevant elements? Is a top-down event boundary generated to ensure that all elements of the event are encoded?

3) The contents of the original event are more likely to be recalled than the second event. Our framework proposes that the original event memory is reactivated and then cycled back to the hippocampus to recreate the theta-gamma code with information about the ongoing event conjoined to it. This means that two traces of the original event are available for retrieval (i.e. the original trace and the conjoined trace), whereas only a single trace of the second event is available (i.e. the conjoined trace). If the conjoined trace were to become distorted or lost, details of the original event may still be recalled from the original trace whereas details of the second event would be lost. As such, memory for the original event should be stronger than that for the second event. In support of this prediction, $A B-B C$ integration paradigms show that memory performance for the first pair is greater than the second pair (Backus et al., 2016; Schlichting et al., 2014). Whether this difference applies to naturalistic events remains an open question however.

4) The number of gamma cycles nested in the hippocampal theta oscillation should increase over the course of an event. As an event unfolds, more details arise. The cell assemblies that code for these new details therefore need to be appended to the theta-gamma code that represents the event. Any additional event information requires additional gamma cycles to 
become nested in the theta oscillation. Heusser and colleagues (2016) have demonstrated such a phenomenon in sequence memory; the addition of an item to an existing sequence produces a shift in metrics of theta-gamma coupling. Our framework would predict that this effect arises regardless of whether the event is later remembered, as the maintenance of the theta-gamma code is essential to comprehending the ongoing event.

5) The original theta-gamma code should be detectable during the unfolding of the second event. Our framework posits that the original event is rapidly reactivated and reconstructed within the hippocampus when a related event begins. Therefore, we speculate that the original theta-gamma code should be detected during the second event. Indeed, previous work has demonstrated that the unique aspect of one pair (i.e. A of pair $A B$ ) is reactivated when the individual is cued with the second pair (i.e. pair BC; Zeithamova et al., 2012). While this paradigm focused on time windows after the pairs $A B$ and $B C$ had been associated, we would postulate that the unique aspect of pair $A B$ is also present during the learning of $B C$, demonstrating that the original event is reactivated during the second event.

\section{Relating to spatial navigation and cognitive maps}

In the previous section, we took a rather narrow view of hippocampal function. Here, we discuss our ideas of event conjunction in the context of other cognitive phenomena that the hippocampus has been linked to.

For every paper linking the hippocampus to episodic memory, there is another linking the hippocampus to spatial navigation. While navigation and memory were once regarded as dissimilar cognitive functions, opinions now favour the idea that the two highly overlap (e.g. Buzsáki \& Moser, 2013). Buzsáki and Moser proposed that the neuronal mechanisms which support spatial navigation are the same mechanisms that support episodic memory; these mechanisms link spatial landmarks to facilitate navigation, and they link objects, locations and concepts to facilitate episodic memory. We believe that our framework conforms to this viewpoint. Indeed, the central tenets of our framework are intimately linked to the neural correlates of spatial navigation. For example, numerous studies have demonstrated that place cells lock to hippocampal theta phase in a temporally-ordered manner to produce a coherent representation of a spatial environment (Dragoi \& Buzsáki, 2006; Skaggs, McNaughton, Wilson, \& Barnes, 1996). Our proposal parallels this by suggesting individual elements of an event (perhaps represented by concept cells; Quiroga, Reddy, Kreiman, Koch, \& Fried, 2005) lock to hippocampal theta phase in an ordered manner to produce a coherent representation of an unfolding event. Whether place sequences can be conjoined in the same manner as we have proposed for events remains an open question. Nevertheless, acknowledging the overlap between event memory and spatial navigation may help address the more complex questions generated by our framework.

An alternative view suggests that spatial navigation and episodic memory are specific forms of a cognitive map. In short, a cognitive map serves to organise and relate experience to influence behaviour across all cognitive domains (Tolman, 1948). Episodic memory sees the traversal of this cognitive map through mental space; spatial navigation sees the traversal of this cognitive map through physical space (Schiller et al., 2015). The hippocampus has long been linked to the concept of the cognitive map. Indeed, map-like relational links across space (Schiller et al., 2015), time (Schapiro, Kustner, \& Turk-Browne, 2012) and social networks (Tavares et al., 2015) are highly dependent on the hippocampus. Of key relevance here, the event models (i.e. representations of ongoing events) frequently discussed in theories of event segmentation share a remarkably high degree of conceptual similarity with the cognitive map. Both refer to internally generated multimodal models that help the individual to comprehend the situation they find themselves in. The key difference is that event 
models are seen to be discarded at event boundaries and replaced with new models, whereas cognitive maps are subject to updating with new information about the change in situational context. Our proposed framework bridges the gap as we describe how the hippocampus can support event models and, critically, relate these event models to previously encoded models. In our opinion, the conceptual idea of a network of interconnected event models is near-indistinguishable from the idea of the cognitive map.

\section{Conclusion}

Episodic memories are carved out of continuous experience through event segmentation. Traditional theories of event segmentation view every event, and therefore every memory, as unique. However, this contradicts our behavioural and electrophysiological understanding of human episodic memory. Here, we have proposed the 'event conjunction framework'. We propose that two unique events which share a commonality can be integrated online (i.e. as the second event unfolds). On an electrophysiological level, we envisage this phenomenon depending on theta-gamma coupling within the hippocampus: elements of an event are represented within individual gamma cycles, with the phase of the theta cycle dictating the start and end of an event. During event conjunction, the thetagamma code that represents a previously-encoded event is reconstructed within the hippocampus and new details from the currently-unfolding event are appended to the code. While some of the central tenets of this framework still require direct empirical investigation, our reinterpretation of existing results provides reassuring support for the framework.

\section{Acknowledgements}

Thanks to (in alphabetical order) Simon Hanslmayr, Catarina Sanches Ferreria and Anna Schapiro for their helpful comments on an earlier draft of this manuscript.

\section{References}

Axmacher, N., Henseler, M. M., Jensen, O., Weinreich, I., Elger, C. E., Fell, J., ... Weinreich, I. (2010). Cross-frequency coupling supports multi-item working memory in the human hippocampus. Proceedings of the National Academy of Sciences, 107(7), 3228-3233. http://doi.org/10.1073/pnas.0911531107

Axmacher, N., Mormann, F., Fernández, G., Elger, C. E., \& Fell, J. (2006). Memory formation by neuronal synchronization. Brain Research Reviews, 52(1), 170-182. http://doi.org/10.1016/j.brainresrev.2006.01.007

Backus, A. R., Schoffelen, J.-M., Szebényi, S., Hanslmayr, S., \& Doeller, C. F. (2016). Hippocampalprefrontal theta oscillations support memory integration. Current Biology, 450-457. http://doi.org/10.1016/j.cub.2015.12.048

Bahramisharif, A., Jensen, O., Jacobs, J., \& Lisman, J. (2018). Serial representation of items during working memory maintenance at letter-selective cortical sites. PLoS Biology, 171660. http://doi.org/10.1101/171660

Baldassano, C., Chen, J., Zadbood, A., Pillow, J. W., Hasson, U., \& Norman, K. A. (2017). Discovering event structure in continuous narrative perception and memory. Neuron, 95(3), 709-721.e5. http://doi.org/10.1016/j.neuron.2017.06.041

Ben-Yakov, A., \& Dudai, Y. (2011). Constructing realistic engrams: Poststimulus activity of hippocampus and dorsal striatum predicts subsequent episodic memory. Journal of Neuroscience, 31(24), 9032-9042. http://doi.org/10.1523/JNEUROSCI.0702-11.2011

Ben-Yakov, A., \& Henson, R. (2018). The hippocampal film-editor: sensitivity and specificity to event 
boundaries in continuous experience. The Journal of Neuroscience, 38(47), 273409. http://doi.org/10.1523/JNEUROSCI.0524-18.2018

Bi, G., \& Poo, M. (1998). Synaptic modifications in cultured hippocampal neurons: Dependence on spike timing, synaptic strength, and postsynaptic cell type. Journal of Neuroscience, 18(24), 1-9. http://doi.org/10.1038/25665

Buzsáki, G., \& Moser, E. I. (2013). Memory, navigation and theta rhythm in the hippocampalentorhinal system. Nature Neuroscience, 16(2), 130-138. http://doi.org/10.1038/nn.3304

Carr, M. F., Jadhav, S. P., \& Frank, L. M. (2011). Hippocampal replay in the awake state: A potential substrate for memory consolidation and retrieval. Nature Neuroscience, 14(2), 147-153. http://doi.org/10.1038/nn.2732

Clewett, D., Dubrow, S., \& Davachi, L. (2019). Transcending time in the brain: How event memories are constructed from experience. Hippocampus, (January), 1-22. http://doi.org/10.1002/hipo.23074

Clouter, A., Shapiro, K. L., \& Hanslmayr, S. (2017). Theta phase synchronization is the glue that binds human associative memory. Current Biology, 1-6. http://doi.org/10.1016/j.cub.2017.09.001

Colgin, L. L. (2015). Theta-gamma coupling in the entorhinal-hippocampal system. Current Opinion in Neurobiology, 31, 45-50. http://doi.org/10.1016/j.conb.2014.08.001

Colgin, L. L., \& Moser, E. I. (2010). Gamma oscillations in the hippocampus. Physiology, 25(5), 319329. http://doi.org/10.1152/physiol.00021.2010

Corkin, S. (2002). What's new with the amnesic patient H. M.? Nature Rev. Neurosci., 3(February), 153-160. Retrieved from http://dx.doi.org/10.1038/nrn726

Craik, F. I. M. (1970). The fate of primary memory items in free recall. Journal of Verbal Learning and Verbal Behavior, 9(2), 143-148. http://doi.org/10.1016/S0022-5371(70)80042-1

Davachi, L. (2006). Item, context and relational episodic encoding in humans. Current Opinion in Neurobiology, 16(6), 693-700. http://doi.org/10.1016/j.conb.2006.10.012

Dragoi, G., \& Buzsáki, G. (2006). Temporal encoding of place sequences by hippocampal cell assemblies. Neuron, 50(1), 145-157. http://doi.org/10.1016/j.neuron.2006.02.023

DuBrow, S., \& Davachi, L. (2013). The influence of context boundaries on memory for the sequential order of events. Journal of Experimental Psychology: General, 142(4), 1277-1286. http://doi.org/10.1037/a0034024

DuBrow, S., \& Davachi, L. (2016). Temporal binding within and across events. Neurobiology of Learning and Memory, 134, 107-114. http://doi.org/10.1016/j.nlm.2016.07.011

Ezzyat, Y., \& Davachi, L. (2011). What constitutes an episode in episodic memory? Psychological Science, 22(2), 243-252. http://doi.org/10.1177/0956797610393742

Giovanello, K. S., Schnyer, D., \& Verfaellie, M. (2009). Distinct hippocampal regions make unique contributions to relational memory. Hippocampus, 19(2), 111-117. http://doi.org/10.1002/hipo.20491

Greene, R. L. (1987). Effects of maintenance rehearsal on human memory. Psychological Bulletin, 102(3), 403. Retrieved from https://insights.ovid.com/plbul/198711000/00006823-19871100000005

Griffiths, B. J., Mazaheri, A., Debener, S., \& HansImayr, S. (2016). Brain oscillations track the formation 
of episodic memories in the real world. Neurolmage, 143, 256-266.

http://doi.org/10.1101/042929

Griffiths, B. J., Michelmann, S., Roux, F., Chelvarajah, R., Rollings, D. T., Sawlani, V., ... Hanslmayr, S. (2018). Hippocampal synchrony and neocortical desynchrony cooperate to encode and retrieve episodic memories. BioRxiv, 305698. http://doi.org/10.1101/305698

HansImayr, S., Staresina, B. P., \& Bowman, H. (2016). Oscillations and episodic memory - Addressing the synchronization/desynchronization conundrum. Trends in Neurosciences, 39(1), 16-25. http://doi.org/10.1016/j.tins.2015.11.004

Hasselmo, M. E. (2005). What is the function of hippocampal theta rhythm? - Linking behavioral data to phasic properties of field potential and unit recording data. Hippocampus, 15(7), 936-949. http://doi.org/10.1002/hipo.20116

Heusser, A. C., Poeppel, D., Ezzyat, Y., \& Davachi, L. (2016). Episodic sequence memory is supported by a theta-gamma phase code. Nature Neuroscience, 19(August). http://doi.org/10.1038/nn.4374

Horner, A. J., Bisby, J. A., Wang, A., Bogus, K., \& Burgess, N. (2016). The role of spatial boundaries in shaping long-term event representations. Cognition, 154, 151-164. http://doi.org/10.1016/j.cognition.2016.05.013

Huerta, P. T., \& Lisman, J. E. (1995). Bidirectional synaptic plasticity induced by a single burst during cholinergic theta oscillation in CA1 in vitro. Neuron, 15(5), 1053-1063. http://doi.org/10.1016/0896-6273(95)90094-2

Hyman, J. M., Wyble, B. P., Goyal, V., Rossi, C. A., \& Hasselmo, M. E. (2003). Stimulation in hippocampal region CA1 in behaving rats yields long-term potentiation when delivered to the peak of theta and long-term depression when delivered to the trough. Journal of Neuroscience, 23(37), 11725-31. http://doi.org/23/37/11725 [pii]

Jutras, M. J., Fries, P., \& Buffalo, E. A. (2009). Gamma-Band Synchronization in the Macaque Hippocampus and Memory Formation. Journal of Neuroscience, 29(40), 12521-12531. http://doi.org/10.1523/JNEUROSCI.0640-09.2009

Kerrén, C., Linde-Domingo, J., Hanslmayr, S., \& Wimber, M. (2018). An optimal oscillatory phase for pattern reactivation during memory retrieval. Current Biology, 28(21), 3383-3392.e6. http://doi.org/10.1016/j.cub.2018.08.065

Konkel, A., \& Cohen, N. J. (2009). Relational memory and the hippocampus: Representations and methods. Frontiers in Neuroscience, 3(SEP), 166-174.

http://doi.org/10.3389/neuro.01.023.2009

Koster, R., Chadwick, M. J., Chen, Y., Berron, D., Banino, A., Düzel, E., ... Kumaran, D. (2018). Big-loop recurrence within the hippocampal system supports integration of information across episodes. Neuron, 99(6), 1342-1354.e6. http://doi.org/10.1016/j.neuron.2018.08.009

Lisman, J. E., \& Jensen, O. (2013). The theta-gamma neural code. Neuron, 77(6), 1002-1016. http://doi.org/10.1016/j.neuron.2013.03.007

Long, N. M., \& Kahana, M. J. (2015). Successful memory formation is driven by contextual encoding in the core memory network. Neurolmage, 119, 332-337.

http://doi.org/10.1016/j.neuroimage.2015.06.073

Meunier, G. F., Ritz, D., \& Meunier, J. A. (1972). Rehearsal of individual items in short-term memory. Journal of Experimental Psychology, 95(2), 465-467. http://doi.org/10.1037/h0033657 
Miller, J. F., Neufang, M., Solway, A., Brandt, A., Trippel, M., Mader, I., ... Schulze-Bonhage, A. (2013). Neural activity in human hippocampal formation reveals the spatial context of retrieved memories. Science, 342(6162), 1111-1114. http://doi.org/10.1126/science.1244056

Nadel, L., Hupbach, A., Gomez, R., \& Newman-Smith, K. (2012). Memory formation, consolidation and transformation. Neuroscience and Biobehavioral Reviews, 36, 1640-1645. http://doi.org/10.1016/j.neubiorev.2012.03.001

Nyhus, E., \& Curran, T. (2010). Functional role of gamma and theta oscillations in episodic memory. Neuroscience and Biobehavioral Reviews, 34(7), 1023-1035. http://doi.org/10.1016/j.neubiorev.2009.12.014

Olsen, R. K., Moses, S. N., Riggs, L., \& Ryan, J. D. (2012). The hippocampus supports multiple cognitive processes through relational binding and comparison. Frontiers in Human Neuroscience, 6(May), 146. http://doi.org/10.3389/fnhum.2012.00146

Pajkert, A., Finke, C., Shing, Y. L., Hoffmann, M., Sommer, W., Heekeren, H. R., \& Ploner, C. J. (2017). Memory integration in humans with hippocampal lesions. Hippocampus, 27, 1230-1238. http://doi.org/10.1002/hipo.22766

Parish, G., Hanslmayr, S., \& Bowman, H. (2018). The sync/desync model: How a synchronized hippocampus and a desynchronized neocortex code memories. Journal of Neuroscience, 38(14), 3428-3440. http://doi.org/10.1523/JNEUROSCI.2561-17.2018

Pavlides, C., Greenstein, Y. J., Grudman, M., \& Winson, J. (1988). Long-term potentiation in the dentate gyrus is induced preferentially on the positive phase of $\theta$-rhythm. Brain Research, 439(1-2), 383-387. http://doi.org/10.1016/0006-8993(88)91499-0

Piekema, C., Kessels, R. P. C., Mars, R. B., Petersson, K. M., \& Fernandez, G. (2006). The right hippocampus participates in short-term memory maintenance of object-location associations. Neurolmage, 33(1), 374-382. Retrieved from http://www.sciencedirect.com/science/article/pii/S1053811906006926

Polyn, S. M., Norman, K. A., \& Kahana, M. J. (2009). A context maintenance and retrieval model of organizational processes in free recall. Psychological Review, 116(1), 129-156. http://doi.org/10.1037/a0014420.A

Preston, A. R., \& Eichenbaum, H. (2013). Interplay of hippocampus and prefrontal cortex in memory. Current Biology, 23(17), R764-R773. http://doi.org/10.1016/j.cub.2013.05.041

Quiroga, R. Q., Reddy, L., Kreiman, G., Koch, C., \& Fried, I. (2005). Invariant visual representation by single neurons in the human brain. Nature, 435(7045), 1102-1107.

http://doi.org/10.1038/nature03687

Radvansky, G. A. (2012). Across the event horizon. Current Directions in Psychological Science, 21(4), 269-272. http://doi.org/10.1177/0963721412451274

Radvansky, G. A., \& Copeland, D. E. (2006). Walking through doorways causes forgetting: Situation models and experienced space. Memory and Cognition, 34(5), 1150-1156. http://doi.org/10.3758/BF03193261

Radvansky, G. A., \& Zacks, J. M. (2017). Event boundaries in memory and cognition. Current Opinion in Behavioral Sciences, 17, 133-140. http://doi.org/10.1016/j.cobeha.2017.08.006

Sargent, J. Q., Zacks, J. M., Hambrick, D. Z., Zacks, R. T., Kurby, C. A., Bailey, H. R., ... Beck, T. M. (2013). Event segmentation ability uniquely predicts event memory. Cognition, 129(2), 241-255. http://doi.org/10.1016/j.cognition.2013.07.002 
Schapiro, A. C., Kustner, L. V., \& Turk-Browne, N. B. (2012). Shaping of object representations in the human medial temporal lobe based on temporal regularities. Current Biology, 22(17), 16221627. http://doi.org/10.1016/j.cub.2012.06.056

Schapiro, A. C., Rogers, T. T., Cordova, N. I., Turk-Browne, N. B., \& Botvinick, M. M. (2013). Neural representations of events arise from temporal community structure. Nature Neuroscience, 16(4), 486-492. http://doi.org/10.1038/nn.3331

Schapiro, A. C., Turk-Browne, N. B., Botvinick, M. M., \& Norman, K. A. (2017). Complementary learning systems within the hippocampus: A neural network modeling approach to recomciling episodic memory with statistical learning. Philosophical Transactions of the Royal Society B, 372. http://doi.org/10.1098/rstb.2016.0049

Schapiro, A. C., Turk-Browne, N. B., Norman, K. A., \& Botvinick, M. M. (2016). Statistical learning of temporal community structure in the hippocampus. Hippocampus, 26(1), 3-8. http://doi.org/10.1002/hipo.22523

Schiller, D., Eichenbaum, H., Buffalo, E. a., Davachi, L., Foster, D. J., Leutgeb, S., \& Ranganath, C. (2015). Memory and space: Towards an understanding of the cognitive map. Journal of Neuroscience, 35(41), 13904-13911. http://doi.org/10.1523/JNEUROSCI.2618-15.2015

Schlichting, M. L., \& Preston, A. R. (2015). Memory integration: Neural mechanisms and implications for behavior. Current Opinion in Behavioral Sciences, 1, 1-8.

http://doi.org/10.1016/j.cobeha.2014.07.005

Schlichting, M. L., Zeithamova, D., \& Preston, A. R. (2014). CA1 subfield contributions to memory integration and inference. Hippocampus, 24(10), 1248-1260. http://doi.org/10.1002/hipo.22310

Sekeres, M. J., Winocur, G., \& Moscovitch, M. (2018). The hippocampus and related neocortical structures in memory transformation. Neuroscience Letters, 680(August 2017), 39-53. http://doi.org/10.1016/j.neulet.2018.05.006

Silva, M., Baldassano, C., \& Fuentemilla, L. (2019). Rapid memory reactivation at movie event boundaries promotes episodic encoding. BioRxiv, 511782. http://doi.org/10.1101/511782

Skaggs, W. E., McNaughton, B. L., Wilson, M. A., \& Barnes, C. A. (1996). Theta phase precession in hippocampal neuronal populations and the compression of temporal sequences. Hippocampus, 6, 149-72.

Sols, I., DuBrow, S., Davachi, L., \& Fuentemilla, L. (2017). Event boundaries trigger rapid memory reinstatement of the prior events to promote their representation in long-term memory. Current Biology, 27(22), 3499-3504.e4. http://doi.org/10.1016/j.cub.2017.09.057

Spalding, K. N., Schlichting, M. L., Zeithamova, D., Preston, A. R., Tranel, D., Duff, M. C., \& Warren, D. E. (2018). Ventromedial prefrontal cortex is necessary for normal associative inference and memory integration. The Journal of Neuroscience, 38(15), 2501-17. http://doi.org/10.1523/JNEUROSCI.2501-17.2018

Speer, N. K., Zacks, J. M., \& Reynolds, J. R. (2007). Human brain activity time-locked to narrative event boundaries. Psychological Science, 18(5), 449-455. http://doi.org/10.1111/j.14679280.2007.01920.x

Staresina, B. P., \& Davachi, L. (2009). Mind the gap: Binding experiences across space and time in the human hippocampus. Neuron, 63(2), 267-276. http://doi.org/10.1016/j.neuron.2009.06.024

Staudigl, T., \& Hanslmayr, S. (2013). Theta oscillations at encoding mediate the context-dependent nature of human episodic memory. Current Biology, 23(12), 1101-1106. 
Tavares, R. M., Mendelsohn, A., Grossman, Y., Williams, C. H., Shapiro, M., Trope, Y., \& Schiller, D. (2015). A Map for Social Navigation in the Human Brain. Neuron, 87(1), 231-243. http://doi.org/10.1016/j.neuron.2015.06.011

Tolman, E. C. (1948). Cognitive maps in rats and men. Psychological Review, 55(4), 189-208.

Tompary, A., \& Davachi, L. (2017). Consolidation promotes the emergence of representational overlap in the hippocampus and medial prefrontal cortex. Neuron, 96(1), 228-241.e5. http://doi.org/10.1016/j.neuron.2017.09.005

Tort, A. B. L., Komorowski, R. W., Manns, J. R., Kopell, N. J., \& Eichenbaum, H. (2009). Theta-gamma coupling increases during the learning of item-context associations. Proceedings of the National Academy of Sciences, 106(49), 20942-20947. http://doi.org/10.1073/pnas.0911331106

Tulving, E. (2002). Episodic memory: from mind to brain. Annual Review of Psychology, 53, 1-25. http://doi.org/10.1146/annurev.psych.53.100901.135114

Unsworth, N., \& Engle, R. W. (2006). Simple and complex memory spans and their relation to fluid abilities: Evidence from list-length effects. Journal of Memory and Language, 54(1), 68-80. http://doi.org/10.1016/j.jml.2005.06.003

Wallenstein, G. V, Eichenbaum, H., \& Hasselmo, M. E. (1998). The hippocampus as an associator of discontiguous events. Trends in Neurosciences, 21(8), 317-323.

Zacks, J. M., Braver, T. S., Sheridan, M. A., Donaldson, D. I., Snyder, A. Z., Ollinger, J. M., ... Raichle, M. E. (2001). Human brain activity time-locked to perceptual event boundaries. Nature Neuroscience, 4, 651-5. http://doi.org/10.1038/88486

Zacks, J. M., \& Swallow, K. M. (2007). Event segmentation. Current Directions in Psychological Science, 16(2), 80-84.

Zeithamova, D., Dominick, A. L., \& Preston, A. R. (2012). Hippocampal and ventral medial prefrontal activation during retrieval-mediated learning supports novel inference. Neuron, 75(1), 168-179. http://doi.org/10.1016/j.neuron.2012.05.010

Zeithamova, D., \& Preston, A. R. (2010). Flexible memories: Differential roles for medial temporal lobe and prefrontal cortex in cross-episode binding. Journal of Neuroscience, 30(44), 14676-14684. http://doi.org/10.1523/JNEUROSCI.3250-10.2010 